\title{
Micro-Droplet Generation and Flow in a Microchannel under the Wall Fluctuation
}

\author{
Yan Pang, Xiang Wang, Zhaomiao Liu \\ College of Mechanical Engineering and Applied Electronics Technology, Beijing University of Technology \\ Pingleyuan 100, Chaoyang District, Beijing, China \\ pangyan@bjut.edu.cn; wxbjut@126.com; lzm@bjut.edu.cn
}

\section{Extended Abstract}

Droplet microfluidics, in which nanoliter to picoliter droplets acted as individual compartments, are common to a diverse array of applications such as analytical chemistry, tissue engineering, microbiology and drug discovery. The droplet generation in a simplified two dimension T-shape microchannel with the main channel width of $50 \mu \mathrm{m}$ and the side channel width of $25 \mu \mathrm{m}$, is simulated to investigate effects of the forced fluctuation of the side wall on the droplet generation and flow. The periodic fluctuations are applied on a length of the side wall in the main channel of the T-junction with the deformation shape of the double-clamped beam acted by the uniform force, which varies with the flow time and fluctuation periods, forms and positions. The relationship of droplet sizes and liquid flow rate follows the scale law from experimental result of normal T-shape microchannel [1]. The fluctuations under most of the conditions expand the distribution range of the droplet size but have a little effect on the average size, while the shape of the fixed side wall changes the average droplet size chiefly. Droplet sizes show a periodic pattern along the relative time when the fluctuation is forced on the side wall near the T-junction. For the droplet size, the applied position shows the effect that the size could be increased when the fluctuation is deformed at the downstream of the junction but the lower nomodispersity also could be induced. The droplet emerging frequency is not varied by the fluctuation of the side wall under the same flow rate and geometry conditions. When the fluctuation period is similar with the droplet emerging period, the droplet size shows a nice stability as the no fluctuation case.

\section{References}

[1] P. Garstecki, et al, "Formation of droplets and bubbles in a microfluidic T-junction-scaling and mechanism of breakup," Lab Chip, vol. 6, no. 3, pp. 437-446, 2006. 